\title{
Development of a L1 tau lepton trigger algorithm for the HL-LHC using the CMS high-granularity calorimeter information
}

Jona Motta* on behalf of the CMS Collaboration

Laboratoire Leprince-Ringuet, CNRS/IN2P3, Ecole Polytechnique, Institut Polytechnique de Paris, Route de Saclay, Palaiseau, France

E-mail: jona.motta@cern.ch

The High-Luminosity LHC will open an unprecedented window on the weak-scale nature of the universe, providing high-precision measurements of the Standard Model (SM) [1] as well as searches for new physics beyond the SM [2]. The CMS Collaboration is planning to replace entirely its trigger and data acquisition systems to match this ambitious physics program. Efficiently collecting datasets in Phase 2 will be a challenging task, given the harsh environment of 200 proton-proton interactions per LHC bunch crossing. The already challenging implementation of an efficient tau lepton trigger will become, in such conditions, an even crucial and harder task; especially interesting will be the case of hadronically decaying taus. To this end, the foreseen high-granularity endcap calorimeter (HGCAL), and the astonishing amount of information it will provide, play a key role in the design of the new online Level-1 (L1) triggering system. In these proceedings, the development of a L1 trigger for hadronically decaying taus based on the sole information from the HGCAL detector will be presented. Some novel ideas for a L1 trigger based on machine learning techniques that can be implemented in FPGA firmware will be presented, as well as the expected performance of the new trigger algorithm based on simulated collision data of the HL-LHC.

The Ninth Annual Conference on Large Hadron Collider Physics - LHCP2021

7-12 June 2021

Online

${ }^{*}$ Speaker 


\section{Introduction}

The High-Luminosity LHC (HL-LHC) phase, in which an instantaneous luminosity of $5 \times 10^{34}$ $\mathrm{cm}^{-2} \mathrm{~s}^{-1}$ is expected, will start in 2027; these intense running conditions result in unprecedented levels of radiation and pileup (PU), and therefore require a major upgrade of the CMS experimental apparatus [3]. This is referred to as "Phase 2 Upgrade" [4] in which, among other things, the trigger and data acquisition system, as well as the endcap calorimeter will be fully replaced in order to increase performances and exploit the full physics potential [5, 6].

The High Granularity Calorimeter (HGCAL) [7] is a 5-dimension (3D position, energy, and time) sampling calorimeter divided into two compartments: a silicon-based electromagnetic one, and a silicon-based plus scintillator tiles hadronic one. This configuration allows very high granularity to be achieved both longitudinally and transversely.

The new trigger system remains organised in two levels, with a hardware Level-1 (L1) trigger and a software High Level Trigger. The L1 trigger is planned to implement custom processor boards and state-of-the-art FPGAs, as well as high-speed optical links to facilitate data aggregation and a highly modular architecture. The key features of the L1 trigger will be the implementation of the correlator trigger where higher level particle flow based variables can be computed benefiting from the first introduction of fully reconstructed tracks at L1 and the HGCAL information.

\section{The HGCAL L1 hadronic tau trigger algorithm}

The development of a dedicated hadronically decaying $\tau_{h}$ trigger has shown great benefits during Run 2 with the observation of $\mathrm{H} \rightarrow \tau \tau$. Inspired by the Phase-1 $\tau$ trigger algorithm, a specific HGCAL algorithm would be crucial to expand the geometrical acceptance in searches for small production cross section processes like $\mathrm{HH} \rightarrow \mathrm{bb} \tau \tau$.

The algorithm presented in the following targets the reconstruction of hadronically decaying $\tau$ leptons $\left(\tau_{h}\right)$ detected by HGCAL; it takes as input the 3D-clusters produced by the HGCAL trigger primitive generator (TPG). Various observables (referred to as shape variables), conveying shape information of the shower created by the $\tau_{h}$ decay products, are also computed by the TPG and exploited to precisely reconstruct the $\tau$-induced signal at trigger level and aid its identification. The algorithm is divided into four steps: seeding, PU rejection, calibration, and decay mode sorting.

As a starting point of the algorithm development, it is assumed that a single 3D-cluster (with a minimal requirement of $\mathrm{E}_{T} \geq 4 \mathrm{GeV}$ ) is associated with the $\tau$-induced shower; the assumption is valid as a single $3 \mathrm{D}$-cluster contains the complete $\tau_{h}$ energy deposit in $\approx 90 \%$ of the cases. Moreover, the selection of a single cluster leads to better pileup rejection. The position resolution achieved in this manner is reported in Fig. 1.

The PU rejection consists in applying a cutoff on the output score of a boosted decision tree (BDT) exploiting 17 cluster shape variables; the L1 $\tau_{h}$ candidates passing the $99 \%$ signal efficiency point are retained.

The calibration of the $\tau_{h}$ candidates is achieved in three separate steps. The first is an $\eta$ dependent PU subtraction to account for the pileup contributions mistakenly collected during the clustering; the calibrated energy is expressed as $E_{\mathrm{T}, \text { calib } 1}^{\mathrm{L} 1, \tau}=E_{\mathrm{T} \text {,raw }}^{\mathrm{L} 1, \tau}-\left(a_{1}+b_{1} \cdot\left|\eta^{\mathrm{L} 1, \tau}\right|\right)$, where $a_{1}$ and $b_{1}$ are calculated via a linear regression with $\left(p_{\mathrm{T}}^{\text {gen }}-E_{\mathrm{T} \text {,raw }}\right)$ as target. The second step accounts 

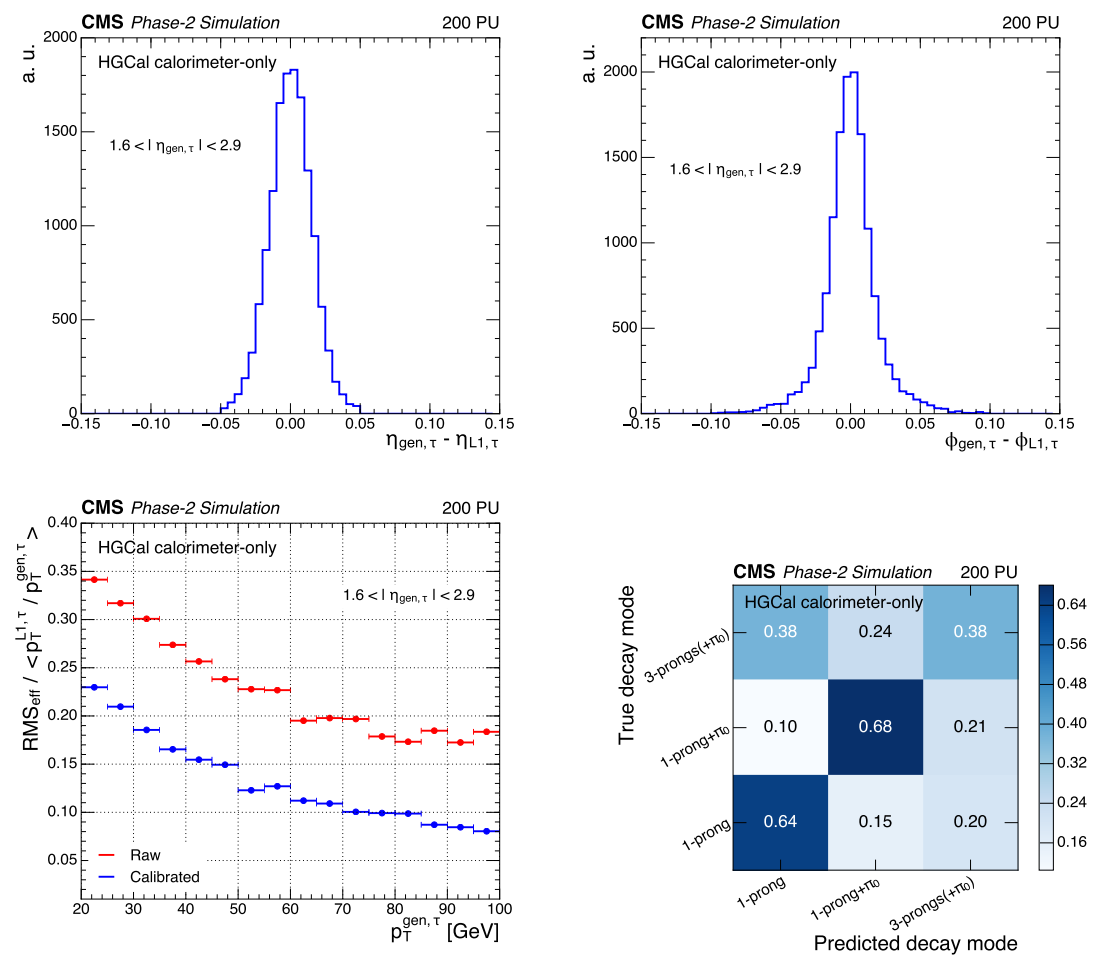

Figure 1: $\eta$ (top left) and $\phi$ (top right) resolutions calculated as the difference in position between the generated visible $\tau_{h}$ and the $\tau_{h} \mathrm{~L} 1$ candidate. Resolution in bins of $p_{\mathrm{T}}$ of the L1 $\tau_{h}$ candidates as a function of to the generated $\tau_{h} p_{\mathrm{T}}$ (bottom left). Confusion matrix between predicted and generated $\tau_{h}$ decay modes. All results are obtained with di- $\tau$ MC samples with 200 average pileup interactions. [8]

for the correction of the different response of HGCAL to the different decay modes of the $\tau_{h}$; this is done with a BDT trained on 18 shape variables and having $p_{\mathrm{T}}^{g e n} / E_{\mathrm{T}, \mathrm{calib} 1}$ as target. The calibrated energy is expressed as $E_{\mathrm{T}, \text { calib2 }}^{\mathrm{L} 1, \tau}=c_{2} \cdot E_{\mathrm{T}, \mathrm{calib} 1}^{\mathrm{L} 1, \tau}$, where $c_{2}$ is calculated by the BDT. Finally, an energy dependent correction is applied; the calibrated energy is expressed as $E_{\mathrm{T}, \text { calib3 }}^{\mathrm{L} 1, \tau}=c_{3} \cdot E_{\mathrm{T}, \text { calib2 }}^{\mathrm{L} 1, \tau}$, where $c_{3}=1 / \sum_{i=0}^{4} k_{3, i} \cdot\left[\log \left(E_{\mathrm{T}, \mathrm{calib} 2}^{\mathrm{L} 1, \tau}\right)\right]^{i}$, and $k_{3, i}$ are calculated by linear regression. The strong impact of the calibration can be seen in Fig. 1.

The identification of the different hadronic decay modes and the consequent categorization is achieved by a random forest classifier exploiting 18 shape variables; the confusion matrix showing the performance of the sorting is given in Fig. 1.

\section{Performance of the algorithm}

The performance is obtained from simulated di- $\tau_{h}$ events with an average of 200 pileup events. The trigger efficiency of a single $\mathrm{L} 1 \tau_{h}$ as a function of its generated visible $p_{\mathrm{T}}$ is shown in Fig. 2, on the top left for different energy thresholds and on the top right for a fixed energy threshold separating the possible decay modes. The curves show a sharp turn-on shape that plateaus at $100 \%$ efficiency for relatively low energy values; the worse selection for multiple prong decays with respect to single prong ones is due to the better reconstruction and identification of the latter. 
The second performance figure of merit is the estimation of the rate associated to the L1 single- $\tau_{h}$ trigger; it is evaluated with simulated neutrino events and it is calculated as a function of the offline threshold. The single- $\tau_{h}$ rates as a function of the offline threshold are reported in Fig. 2 (bottom); the values of the rate are currently too high for a feasible implementation but they can be effectively reduced including isolation requirements on the $\mathrm{L} 1 \tau_{h}$ candidates. Further reduction can be achieved by matching the clusters with charged tracks.

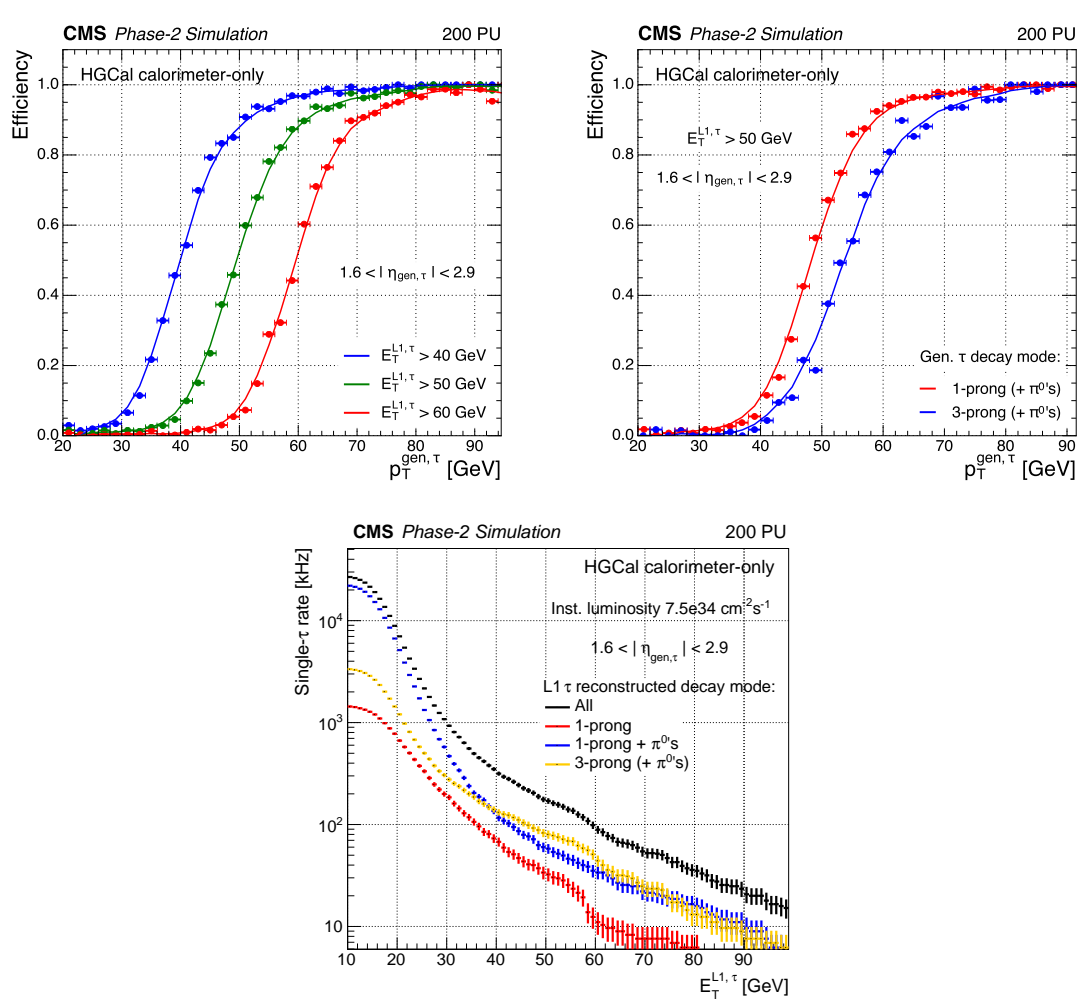

Figure 2: $\mathrm{L} 1 \tau_{h}$ efficiency as a function of the generated visible $\tau_{h} p_{\mathrm{T}}$ for $\mathrm{L} 1$ thresholds of $40 \mathrm{GeV}, 50 \mathrm{GeV}$ and $60 \mathrm{GeV}$ (top left), and for different decay modes for a L1 threshold of $50 \mathrm{GeV}$ (top right), both obtained with di- $\tau$ MC samples with 200 average pileup interactions. Single L1 $\tau_{h}$ rates as a function of the L1 $\tau_{h} p_{\mathrm{T}}$ threshold (bottom), obtained with a neutrino MC sample with 200 average pileup interactions [8]

\section{Conclusions and outlook}

The Phase-2 L1 trigger upgrades permit substantial improvement in the implementation of sophisticated machine-learning-based $\tau_{h}$ trigger algorithms. A better knowledge of the shower features allows BDT-based trigger primitive selection and calibration to be implemented. A preliminary analysis shows that the algorithm has a very satisfactory performance, namely: a 19\% energy resolution, a sharp selection efficiency that reaches $100 \%$ at plateau, and the possibility to separate the decay modes at L1 level.

Despite the good performance already achieved, developments of further machine-learningbased methods are ongoing to implement isolation requirements and QCD-jet rejection. Finally, the first firmware implementation of the algorithm is underway. 


\section{References}

[1] P. Azzi et al., Report from Working Group 1: Standard Model physics at the HL-LHC and HE-LHC, CERN Yellow Rep. Monogr. 7 (2019) 1 [1902 . 04070].

[2] X. Cid Vidal et al., Report from Working Group 3: Beyond the Standard Model physics at the HL-LHC and HE-LHC, CERN Yellow Rep. Monogr. 7 (2019) 585 [1812. 07831].

[3] CMS collaboration, The CMS Experiment at the CERN LHC, JINST 3 (2008) S08004.

[4] D. Contardo, M. Klute, J. Mans, L. Silvestris and J. Butler, Technical Proposal for the Phase-II Upgrade of the CMS Detector, Tech. Rep. CMS-TDR-15-02, CERN, Geneva (Jun, 2015).

[5] CMS, ATLAS collaboration, Addendum to the report on the physics at the HL-LHC, and perspectives for the HE-LHC: Collection of notes from ATLAS and CMS, Tech. Rep. CERN-LPCC-2019-01, CERN, Geneva (Dec, 2019), DOI.

[6] CMS COLlaboRATION collaboration, Expected performance of the physics objects with the upgraded CMS detector at the HL-LHC, Tech. Rep. CMS-NOTE-2018-006, CERN, Geneva (Dec, 2018).

[7] CMS collaboration, The Phase-2 Upgrade of the CMS Endcap Calorimeter, Tech. Rep. CMS-TDR-019, CERN, Geneva (Nov, 2017).

[8] CMS collaboration, The phase-2 upgrade of the cms level-1 trigger, Tech. Rep. CMS-TDR-021, CERN, Geneva (Apr, 2020). 